International Journal of Pure and Applied Mathematics

Volume $93 \quad$ No. $3 \quad 2014,427-447$

ISSN: 1311-8080 (printed version); ISSN: 1314-3395 (on-line version)

url: http://www.ijpam.eu

doi: http://dx.doi.org/10.12732/ijpam.v93i3.12

ijpam.eu

\title{
IMPROVED DELAY-RANGE-DEPENDENT STABILITY CRITERIA FOR DISCRETE-TIME LINEAR SYSTEMS WITH INTERVAL TIME-VARYING DELAY AND NONLINEAR PERTURBATIONS
}

\author{
Cheerapong Singkibud ${ }^{1}$, Kanit Mukdasai $^{2} \S$ \\ ${ }^{1,2}$ Department of Mathematics \\ Faculty of Science \\ Khon Kaen University \\ Khon Kaen 40002, THAILAND
}

\begin{abstract}
In this paper, we study the problem of stability analysis for discrete-time linear system with interval time-varying delay and nonlinear perturbations. By constructing a new Lyapunov-Krasovskii functional with triple summation terms, mixed model transformation, Jensen-type summation inequality and utilization of zero equation, new delay-range-dependent asymptotic stability criteria are obtained and formulated in terms of linear matrix inequalities (LMIs). Moreover, we obtain new delay-range-dependent asymptotic stability criteria of discrete-time linear system with interval time-varying delay. Numerical examples are given to demonstrate the effectiveness and less conservativeness of the proposed methods.
\end{abstract}

AMS Subject Classification: 26D15, 34D20, 34K20, 37B25, 37C75, 37D40. Key Words: delay-range-dependent stability criteria, discrete-time linear system, interval time-varying delay, nonlinear perturbations, model transformation, linear matrix inequality

Received: March 1, 2014

(C) 2014 Academic Publications, Ltd.

${ }^{\S}$ Correspondence author url: www.acadpubl.eu 


\section{Introduction}

Time-delay systems exist in many fields such as electric systems, chemical processes systems, networked control systems, telecommunication systems and economical systems. During the past two decades, the problems of robust stability and stabilization analysis for dynamic systems with time delays have been widely investigated by many researchers [1]-[26]. It is well known that nonlinearities, as time delays, may cause instability and poor performance of practical systems. Commonly, stability criteria for dynamic systems with time delay are generally divided into two classes: a delay-independent one and a delay-dependent one. The delay-independent stability criteria tend to be more conservative, especially for a small size delay, such criteria do not give any information on the size of delay. On the other hand, delay-dependent stability criteria are concerned with the size of delay and usually provide a maximal delay size. Recently, a special type of time delay in practical engineering systems, that is interval time-varying delay, is investigated. The characteristic of interval time-varying delay is that time delay can vary in an interval in which the lower bound of delay is not restricted to zero. The typical examples of systems with interval time-varying delay are networked control systems, chemical process and flight systems [10].

Discrete-time systems with state delay have strong background in engineering applications, among which network based control has been well recognized to be a typical example. If the delay is constant in discrete systems, one can transform a delayed system in to a delay-free one by using state augmentation techniques. However, when the delay is large, the augmented system will become much complex and thus difficult to analyze and synthesize [5]. Hence, researchers have focussed on the delay-range-dependent stability and stabilization problems of discrete-time systems with interval time-varying delay and many existing results mainly focus on discrete-time linear delay systems [1]-[12], [14][15], [17]-[23], [25]-[26]. However, most real systems hold nonlinear dynamics. Therefore, researchers have been investigated the delay-range-dependent stability criteria for discrete-time linear systems with interval time-varying delay and nonlinear perturbations [13], [16], [24]. However, the existing results of delay-range-dependent stability criteria do not take into account the presence of nonlinear perturbations uncertainties in the discrete-time delay systems by model transformation.

This paper will focus on the delay-range-dependent stability analysis for discrete-time linear system with interval time-varying delay and nonlinear perturbations. By using the combination of mixed model transformation, Jensen- 
type summation inequality, utilization of zero equation and new LyapunovKrasovskii functional, new delay-range-dependent asymptotic stability criteria are obtained and formulated in terms of LMIs. Then, we can obtain new delayrange-dependent asymptotic stability criteria of discrete-time linear systems with interval time-varying delay. Finally, numerical examples will be given to show the effectiveness of the obtained results.

We introduce some notations, definition and lemmas that will be used throughout the paper. $Z^{+}$denotes the set of all real non-negative numbers; $R^{n}$ denotes the $n$-dimensional space with the vector norm $\|\cdot\| ;\|x\|$ denotes the Euclidean vector norm of $x \in R^{n} ; R^{n \times r}$ denotes the set of $n \times r$ real constant matrices; $A^{T}$ denotes the transpose of the matrix $A ; A$ is symmetric if $A=A^{T}$; $I$ denotes the identity matrix; Matrix $A$ is called semi-positive definite $(A \geq 0)$ if $x^{T} A x \geq 0$, for all $x \in R^{n} ; A$ is positive definite $(A>0)$ if $x^{T} A x>0$ for all $x \neq 0$; Matrix $B$ is called semi-negative definite $(B \leq 0)$ if $x^{T} B x \leq 0$, for all $x \in R^{n} ; B$ is negative definite $(B<0)$ if $x^{T} B x<0$ for all $x \neq 0 ; A>B$ means $A-B>0(B-A<0) ; A \geq B$ means $A-B \geq 0(B-A \leq 0)$.

\section{Problem Formulation and Preliminaries}

Consider the discrete-time linear system with interval time-varying delay and nonlinear perturbations of the form

$$
\begin{aligned}
x(k+1)= & A x(k)+B x(k-h(k))+f(k, x(k)) \\
& +g(k, x(k-h(k))), \\
x(s)= & \phi(s), \quad s \in\left\{-h_{2},-h_{2}+1, \ldots, 0,\right\},
\end{aligned}
$$

where $k \in Z^{+}, x(k) \in R^{n}$ is the state variable and $\phi(s)$ is a initial value at $s$. $A$ and $B \in R^{n \times n}$ are known real constant matrices. $f(k, x(k))$ and $g(k, x(k-h(k)))$ are the nonlinear perturbations with respect to current state $x(k)$ and discrete delay state $x(k-h(k))$, respectively, and are bounded in magnitude:

$$
\begin{aligned}
\|f(k, x(k))\| & \leq \alpha\|x(k)\|, \\
\|g(k, x(k-h(k)))\| & \leq \beta\|x(k-h(k))\|,
\end{aligned}
$$

where $\alpha$ and $\beta$ are given positive real constants. In addition, we assume that the time-varying delay $h(k)$ is upper and lower bounded. It satisfies the following assumption of the form

$$
0<h_{1} \leq h(k) \leq h_{2},
$$


where $h_{1}$ and $h_{2}$ are known positive real constants. Rewrite the system (1) in the following system:

$$
\begin{aligned}
x(k+1)= & x(k)+y(k), \\
y(k)= & {[A+B-I] x(k)-B \sum_{i=k-h(k)}^{k-1} y(i) } \\
& +f(k, x(k))+g(k, x(k-h(k))) .
\end{aligned}
$$

By utilizing the following zero equation, we have

$$
J x(k)-J x(k-h(k))-J \sum_{i=k-h(k)}^{k-1} y(i)=0,
$$

where $J \in R^{n \times n}$ will be chosen to guarantee the asymptotic stability of the system (1)-(2). By (7), system (5)-(6) can be represented by the form

$$
\begin{aligned}
x(k+1)= & x(k)+y(k)+J x(k)-J x(k-h(k)) \\
& -J \sum_{i=k-h(k)}^{k-1} y(i), \\
y(k)= & {[A+B-I] x(k)-B \sum_{i=k-h(k)}^{k-1} y(i) } \\
& +f(k, x(k))+g(k, x(k-h(k))) .
\end{aligned}
$$

Definition 2.1. [18] The system (1)-(2) is said to be asymptotically stable if there exists a positive definite function $V(k, x(k)): Z^{+} \times R^{n} \rightarrow R$ such that

$$
\Delta V(k, x(k))=V(k+1, x(k+1))-V(k, x(k))<0,
$$

along any trajectory of solution for system (1)-(2).

Lemma 2.2. [6] [Schur complement lemma] Given constant symmetric matrices $X, Y$ and $Z$ of appropriate dimensions with $Y>0$, then $X+Z^{T} Y^{-1} Z<0$ if and only if

$$
\left(\begin{array}{cc}
X & Z^{T} \\
Z & -Y
\end{array}\right)<0
$$


Lemma 2.3. [8] For any constant matrix $W \in R^{n \times n}, W=W^{T}>0$, two integers $r_{M}$ and $r_{m}$ satisfying $r_{M} \geq r_{m}$ and vector function $x:\left[r_{m}, r_{M}\right] \rightarrow R^{n}$, the following inequality holds:

$$
\left(\sum_{i=r_{m}}^{r_{M}} x(i)\right)^{T} W\left(\sum_{i=r_{m}}^{r_{M}} x(i)\right) \leq \delta \sum_{i=r_{m}}^{r_{M}} x^{T}(i) W x(i),
$$

where $\delta=r_{M}-r_{m}+1$.

Lemma 2.4. [19] Let $M \in R^{n \times n}$ be a positive-definite matrix, $X_{i} \in$ $R^{n}, i=1,2, \ldots$ If the sums concerned are well defined, then

$$
\begin{aligned}
& \left(\sum_{i=k-M}^{k-N-1} \sum_{j=i}^{k-N-1} X_{j}\right)^{T} M\left(\sum_{i=k-M}^{k-N-1} \sum_{j=i}^{k-N-1} X_{j}\right) \\
& \leq \frac{(M-N)^{2}}{2} \sum_{i=k-M}^{k-N-1} \sum_{j=i}^{k-N-1} X_{j}^{T} M X_{j} .
\end{aligned}
$$

We introduce the following notations for later use.

$$
\prod=\left(\Sigma_{k, l}\right)_{13 \times 13},
$$

where $\quad \Sigma_{k, l}=\Sigma_{l, k}^{T}, \quad k, l=1,2,3, \ldots, 13$,

$$
\begin{aligned}
\Sigma_{1,1}= & L_{1}^{T} A+L_{1}^{T} B-L_{1}^{T}+A^{T} L_{1}+B^{T} L_{1}-L_{1} \\
& +Q+R+T+h_{1} M+N+N^{T}+K+L \\
& +L^{T}+h_{1}^{2} F+C_{1}^{T}+C_{1}+D_{1}^{T}+D_{1} \\
& +E_{1}^{T}+E_{1}+G_{1}^{T}+G_{1}+\epsilon_{1} \alpha^{2} I+2 \hat{P}, \\
\Sigma_{1,2}= & P-L_{1}^{T}+A^{T} L_{2}+B^{T} L_{2}-L_{2}+C_{2}+D_{2} \\
& +E_{2}+G_{2}+\hat{P}, \\
\Sigma_{1,3}= & -N+C_{3}-C_{1}^{T}+D_{3}+E_{3}+G_{3}, \\
\Sigma_{1,4}= & L+C_{4}+D_{4}-D_{1}^{T}+E_{4}+G_{4}-G_{1}^{T}, \\
\Sigma_{1,5}= & C_{5}+D_{5}+E_{5}-E_{1}^{T}+G_{5}-\hat{P}, \\
\Sigma_{1,6}= & h_{1} F+C_{6}-C_{1}^{T}+D_{6}+E_{6}+G_{6}, \\
\Sigma_{1,7}= & C_{7}+D_{7}-D_{1}^{T}+E_{7}+G_{7}, \\
\Sigma_{1,8}= & -L_{1}^{T} B+A^{T} L_{3}+B^{T} L_{3}-L_{3}+C_{8} \\
& +D_{8}+E_{8}-E_{1}^{T}+G_{8}-\hat{P},
\end{aligned}
$$




$$
\begin{aligned}
& \Sigma_{1,9}=C_{9}+D_{9}+E_{9}+G_{9}-G_{1}^{T}, \\
& \Sigma_{1,10}=C_{10}+D_{10}+E_{10}+G_{10} \text {, } \\
& \Sigma_{1,11}=C_{11}+D_{11}+E_{11}+G_{11} \text {, } \\
& \Sigma_{1,12}=C_{12}+D_{12}+E_{12}+G_{12}+L_{1}^{T}, \\
& \Sigma_{1,13}=C_{13}+D_{13}+E_{13}+G_{13}+L_{1}^{T} \text {, } \\
& \Sigma_{2,2}=P-L_{2}^{T}-L_{2}+h_{1}^{2} U+h_{2}^{2} V+h_{2}^{2} W \\
& +\rho^{2} X+h_{1} Y+h_{2} Z+\frac{1}{4} h_{1}^{4} F+\frac{1}{4} \rho^{2} H, \\
& \Sigma_{2,3}=-C_{2}^{T} \text {, } \\
& \Sigma_{2,4}=-D_{2}^{T}-G_{2}^{T}, \\
& \Sigma_{2,5}=-E_{2}^{T}-\hat{P} \text {, } \\
& \Sigma_{2,6}=-C_{2}^{T} \text {, } \\
& \Sigma_{2,7}=-D_{2}^{T} \\
& \Sigma_{2,8}=-L_{2}^{T} B-L_{3}-E_{2}^{T}-\hat{P}, \\
& \Sigma_{2,9}=-G_{2}^{T} \text {, } \\
& \Sigma_{2,10}=\Sigma_{2,11}=0 \text {, } \\
& \Sigma_{2,12}=L_{2}^{T} \text {, } \\
& \Sigma_{2,13}=L_{2}^{T} \text {, } \\
& \Sigma_{3,3}=-Q+S-H-C_{3}^{T}-C_{3} \text {, } \\
& \Sigma_{3,4}=-C_{4}-D_{3}^{T}-G_{3}^{T} \text {, } \\
& \Sigma_{3,5}=-C_{5}-E_{3}^{T} \text {, } \\
& \Sigma_{3,6}=-C_{6}-C_{3}^{T}, \\
& \Sigma_{3,7}=-C_{7}-D_{3}^{T} \text {, } \\
& \Sigma_{3,8}=-C_{8}-E_{3}^{T} \text {, } \\
& \Sigma_{3,9}=-C_{9}-G_{3}^{T}, \\
& \Sigma_{3,10}=-C_{10} \text {, } \\
& \Sigma_{3,11}=H-C_{11} \text {, } \\
& \Sigma_{3,12}=-C_{12} \text {, } \\
& \Sigma_{3,13}=-C_{13} \text {, } \\
& \Sigma_{4,4}=-R-S-D_{4}^{T}-D_{4}-G_{4}^{T}-G_{4} \text {, } \\
& \Sigma_{4,5}=-D_{5}-E_{4}^{T}-G_{5} \text {, } \\
& \Sigma_{4,6}=-C_{4}^{T}-D_{6}-G_{6} \text {, }
\end{aligned}
$$




$$
\begin{aligned}
& \Sigma_{4,7}=-D_{7}-D_{4}^{T}-G_{7}, \\
& \Sigma_{4,8}=-D_{8}-E_{4}^{T}-G_{8} \text {, } \\
& \Sigma_{4,9}=-D_{9}-G_{9}-G_{4}^{T} \text {, } \\
& \Sigma_{4,10}=-D_{10}-G_{10} \text {, } \\
& \Sigma_{4,11}=-D_{11}-G_{11} \text {, } \\
& \Sigma_{4,12}=-D_{12}-G_{12} \text {, } \\
& \Sigma_{4,13}=-D_{13}-G_{13} \text {, } \\
& \Sigma_{5,5}=-T-H-E_{5}^{T}-E_{5}+\epsilon_{1} \beta^{2} I, \\
& \Sigma_{5,6}=-C_{5}^{T}-E_{6} \text {, } \\
& \Sigma_{5,7}=-D_{5}^{T}-E_{7}, \\
& \Sigma_{5,8}=-E_{8}-E_{5}^{T} \text {, } \\
& \Sigma_{5,9}=-E_{9}-G_{5}^{T} \text {, } \\
& \Sigma_{5,10}=H-E_{10} \text {, } \\
& \Sigma_{5,11}=-E_{11} \text {, } \\
& \Sigma_{5,12}=-E_{12} \text {, } \\
& \Sigma_{5,13}=-E_{13} \text {, } \\
& \Sigma_{6,6}=-U-F-C_{6}^{T}-C_{6}, \\
& \Sigma_{6,7}=-C^{7}-D_{6}^{T}, \\
& \Sigma_{6,8}=-C^{8}-E_{6}^{T}, \\
& \Sigma_{6,9}=-C_{9}-G_{6}^{T}, \\
& \Sigma_{6,10}=-C_{10} \text {, } \\
& \Sigma_{6,11}=-C_{11} \text {, } \\
& \Sigma_{6,12}=-C_{12} \text {, } \\
& \Sigma_{6,13}=-C_{13} \text {, } \\
& \Sigma_{7,7}=-V-D_{7}^{T}-D_{7} \text {, } \\
& \Sigma_{7,8}=-D_{8}-E_{7}^{T}, \\
& \Sigma_{7,9}=-D_{9}-G_{7}^{T}, \\
& \Sigma_{7,10}=-D_{10} \text {, } \\
& \Sigma_{7,11}=-D_{11} \text {, } \\
& \Sigma_{7,12}=-D_{12} \text {, } \\
& \Sigma_{7,13}=-D_{13} \text {, } \\
& \Sigma_{8,8}=-L_{3}^{T} B-B^{T} L_{3}-W-E_{8}^{T}-E_{8},
\end{aligned}
$$




$$
\begin{aligned}
& \Sigma_{8,9}=-E_{9}-G_{8}^{T}, \\
& \Sigma_{8,10}=-E_{10}, \\
& \Sigma_{8,11}=-E_{11} \text {, } \\
& \Sigma_{8,12}=-E_{12}+L_{3}^{T}, \\
& \Sigma_{8,13}=-E_{13}+L_{3}^{T}, \\
& \Sigma_{9,9}=-X-G_{9}^{T}-G_{9} \text {, } \\
& \Sigma_{9,10}=-G_{10} \text {, } \\
& \Sigma_{9,11}=-G_{11} \text {, } \\
& \Sigma_{9,12}=-G_{12} \text {, } \\
& \Sigma_{9,13}=-G_{13} \text {, } \\
& \Sigma_{10,10}=-H \text {, } \\
& \Sigma_{11,11}=-H \text {, } \\
& \Sigma_{12,12}=-\epsilon_{1} I \text {, } \\
& \Sigma_{13,13}=-\epsilon_{2} I \text {, } \\
& \rho=h_{2}-h_{1} \text {, } \\
& \alpha(k)=h(k)-h_{1}, \\
& \beta(k)=h_{2}-h(k) \text {, } \\
& \psi(k)=\frac{1}{\alpha(k)}\left[\sum_{i=k-h(k)}^{k-h_{1}-1} x(i)\right\rfloor, \\
& \phi(k)=\frac{1}{\beta(k)}\left[\sum_{i=k-h_{2}}^{k-h(k)-1} x(i)\right\rfloor, \\
& \hat{P}=P J .
\end{aligned}
$$

\section{Delay-Range-Dependent Stability Criteria}

Theorem 3.1. The system (1)-(2) is asymptotically stable, if there exist positive definite symmetric matrices $P, Q, R, S, T, U, V$, $W, X, Y, Z$, any appropriate dimensional matrices $L_{w}, C_{j}, D_{j}, E_{j}, G_{j}$, $M, N, K, L, J, w=1,2,3, j=1,2, \ldots, 13$ and positive real constants $\epsilon_{1}$ and $\epsilon_{2}$ such that the following symmetric linear matrix inequalities hold:

$$
\prod<0
$$




$$
\begin{aligned}
& \left(\begin{array}{cc}
M & N \\
N^{T} & Y
\end{array}\right) \geq 0 \\
& \left(\begin{array}{cc}
K & L \\
L^{T} & Z
\end{array}\right) \geq 0 .
\end{aligned}
$$

Proof. Consider the following Lyapunov-Krasovskii function for system (8)-(9) of the form

$$
V(k)=\sum_{i=1}^{6} V_{i}(k)
$$

where

$$
\begin{aligned}
& V_{1}(k)=x^{T}(k) P x(k), \\
& V_{2}(k)=\sum_{i=k-h_{1}}^{k-1} x^{T}(i) Q x(i)+\sum_{i=k-h_{2}}^{k-1} x^{T}(i) R x(i) \\
& +\sum_{i=k-h_{2}}^{k-h_{1}-1} x^{T}(i) S x(i)+\sum_{i=k-h(k)}^{k-1} x^{T}(i) T x(i), \\
& V_{3}(k)=h_{1} \sum_{j=-h_{1}}^{-1} \sum_{i=k+j}^{k-1} y^{T}(i) U y(i) \\
& +h_{2} \sum_{j=-h_{2}}^{-1} \sum_{i=k+j}^{k-1} y^{T}(i) V y(i) \\
& +h_{2} \sum_{j=-h_{2}}^{-1} \sum_{i=k+j}^{k-1} y^{T}(i) W y(i) \\
& V_{4}(k)=\rho \sum_{j=-h_{2}}^{-h_{1}-1} \sum_{i=k+j}^{k-1} y^{T}(i) X y(i), \\
& V_{5}(k)=\sum_{j=-h_{1}+1}^{0} \sum_{i=k-1+j}^{k-1} y^{T}(i) Y y(i) \\
& +\sum_{j=-h_{2}+1}^{0} \sum_{i=k-1+j}^{k-1} y^{T}(i) Z y(i)
\end{aligned}
$$




$$
\begin{aligned}
V_{6}(k)= & \frac{h_{1}^{2}}{2} \sum_{i=-h_{1}}^{-1} \sum_{j=i}^{0} \sum_{l=k+j}^{k-1} y^{T}(l) F y(l) \\
& +\frac{1}{2} \sum_{i=k-h_{2}}^{k-h_{1}-1} \sum_{j=i}^{k-h_{1}-1} \sum_{l=j}^{k-h_{1}-1} y^{T}(l) H y(l) .
\end{aligned}
$$

Evaluating the forward deference of $V(k)$, it is defined as

$$
\Delta V(k)=\sum_{i=1}^{6} \Delta V_{i}(k) .
$$

Let us define for $i=1,2, \ldots, 6$,

$$
\Delta V_{i}(k)=V_{i}(k+1)-V_{i}(k) .
$$

Taking the forward deference of $V_{1}(k)$ and $V_{2}(k)$, the increments of $V_{1}(k)$ and $V_{2}(k)$ are

$$
\begin{aligned}
\Delta V_{1}(k)= & x^{T}(k+1) P x(k+1)-x^{T}(k) P x(k) \\
= & {\left[x^{T}(k)+y^{T}(k)\right] P[x(k)+y(k)+J x(k)} \\
& \left.-J x(k-h(k))-J \sum_{i=k-h(k)}^{k-1} y(i)\right] \\
& +\left[x^{T}(k) J^{T}-x^{T}(k-h(k)) J^{T}\right. \\
& \left.-\sum_{i=k-h(k)}^{k-1} y^{T}(i) J^{T}\right] P[x(k)+y(k)] \\
& +2 x^{T}(k) L_{1}^{T}[-y(k)+[A+B-I] x(k) \\
& \left.-B \sum_{i=k-h(k)}^{k-1} y(i)+f(k, x(k))+g(k, x(k-h(k)))\right] \\
& +2 y^{T}(k) L_{2}^{T}[-y(k)+[A+B-I] x(k) \\
& \left.-B \sum_{i=k-h(k)}^{k-1} y(i)+f(k, x(k))+g(k, x(k-h(k)))\right]
\end{aligned}
$$




$$
\begin{aligned}
& +2 \sum_{i=k-h(k)}^{k-1} y(i)^{T} L_{3}^{T}[-y(k)+[A+B-I] x(k) \\
& \left.-B \sum_{i=k-h(k)}^{k-1} y(i)+f(k, x(k))+g(k, x(k-h(k)))\right] \\
\Delta V_{2}(k)= & x^{T}(k) Q x(k)-x^{T}\left(k-h_{1}\right) Q x\left(k-h_{1}\right) \\
& +x^{T}(k) R x(k)-x^{T}\left(k-h_{2}\right) R x\left(k-h_{2}\right) \\
& +x^{T}\left(k-h_{1}\right) S x\left(k-h_{1}\right)-x^{T}\left(k-h_{2}\right) S x\left(k-h_{2}\right) \\
& +x^{T}(k) T x(k)-x^{T}(k-h(k)) T x(k-h(k)) .
\end{aligned}
$$

By Lemma 2.3, the increments of $V_{3}(k)$ and $V_{4}(k)$ are easily computed as

$$
\begin{aligned}
\Delta V_{3}(k)= & h_{1}^{2} y^{T}(k) U y(k)-h_{1} \sum_{i=k-h_{1}}^{k-1} y^{T}(i) U y(i) \\
& +h_{2}^{2} y^{T}(k) V y(k)-h_{2} \sum_{i=k-h_{2}}^{k-1} y^{T}(i) V y(i) \\
& +h_{2}^{2} y^{T}(k) W y(k)-h_{2} \sum_{i=k-h_{2}}^{k-1} y^{T}(i) W y(i) \\
\leq & h_{1}^{2} y^{T}(k) U y(k)-\left(\sum_{i=k-h_{1}}^{k-1} y(i)\right)^{T} U\left(\sum_{i=k-h_{1}}^{k-1} y(i)\right) \\
& +h_{2}^{2} y^{T}(k) V y(k)-\left(\sum_{i=k-h_{2}}^{k-1} y(i)\right)^{T} V\left(\sum_{i=k-h_{2}}^{k-1} y(i)\right) \\
& +h_{2}^{2} y^{T}(k) W y(k)-\left(\sum_{i=k-h(k)}^{k-1} y(i)\right)^{T} W\left(\sum_{i=k-h}^{k-1} y(i)\right), \\
\Delta V_{4}(k)= & \left.\rho^{2} y^{T}(k) X y(k)-\rho \sum_{i=k-h_{2}}^{k-h_{1}-1} y(i)\right)^{T} X\left(\sum_{i=k-h_{2}}^{k-h_{1}-1} y(i)\right), X y(i) \\
&
\end{aligned}
$$


Taking the forward deference of $V_{5}(k)$ yields

$$
\begin{aligned}
\Delta V_{5}(k)= & h_{1} y^{T}(k) Y y(k)-\sum_{i=-h_{1}+1}^{0} y^{T}(k-1+i) Y y(k-1+i) \\
& +h_{2} y^{T}(k) Z y(k)-\sum_{i=-h_{2}+1}^{0} y^{T}(k-1+i) Z y(k-1+i) .
\end{aligned}
$$

By (12) and (13), it is easy to see that

$$
\begin{aligned}
& 2 x^{T}(k) N \sum_{i=-h_{1}+1}^{0} y(k-1+i) \\
& +\sum_{i=-h_{1}+1}^{0} y^{T}(k-1+i) Y y(k-1+i) \\
& +h_{1} x^{T}(k) M x(k)=\sum_{i=-h_{1}+1}^{0}\left(\begin{array}{c}
x(k) \\
y(k-1+i)
\end{array}\right)^{T} \\
& \left(\begin{array}{cc}
M & N \\
N^{T} & Y
\end{array}\right)\left(\begin{array}{c}
x(k) \\
y(k-1+i)
\end{array}\right) \geq 0, \\
& 2 x^{T}(k) L \sum_{i=-h_{1}+1}^{0} y(k-1+i) \\
& +\sum_{i=-h_{2}+1}^{0} y^{T}(k-1+i) Z y(k-1+i) \\
& +h_{2} x^{T}(k) K x(k)=\sum_{i=-h_{2}+1}^{0}\left(\begin{array}{c}
x(k) \\
y(k-1+i)
\end{array}\right)^{T} \\
& \left(\begin{array}{ll}
K & L \\
L^{T} & Z
\end{array}\right)\left(\begin{array}{c}
x(k) \\
y(k-1+i)
\end{array}\right) \geq 0 .
\end{aligned}
$$

From (22) and (23), we can obtain

$$
\begin{gathered}
-\sum_{i=-h_{1}+1}^{0} y^{T}(k-1+i) Y y(k-1+i) \\
\leq h_{1} x^{T}(k) M x(k)+2 x^{T}(k) N \sum_{i=-h_{1}+1}^{0} y(k-1+i)
\end{gathered}
$$




$$
\begin{aligned}
= & h_{1} x^{T}(k) M x(k)+2 x^{T}(k) N x(k) \\
& -2 x^{T}(k) N x\left(k-h_{1}\right),
\end{aligned}
$$

and we have

$$
\begin{aligned}
& -\sum_{i=-h_{2}+1}^{0} y^{T}(k-1+i) Z y(k-1+i) \\
\leq & h_{2} x^{T}(k) K x(k)+2 x^{T}(k) L \sum_{i=-h_{2}+1}^{0} y(k-1+i) \\
= & h_{2} x^{T}(k) K x(k)+2 x^{T}(k) L x(k) \\
& -2 x^{T}(k) L x\left(k-h_{2}\right) .
\end{aligned}
$$

Therefore, we conclude that

$$
\begin{aligned}
\Delta V_{5}(k) \leq & h_{1} y^{T}(k) Y y(k)+h_{1} x^{T}(k) M x(k) \\
& +2 x^{T}(k) N x(k)-2 x^{T}(k) N x\left(k-h_{1}\right) \\
& +h_{2} y^{T}(k) Z y(k)+h_{2} x^{T}(k) K x(k) \\
& +2 x^{T}(k) L x(k)-2 x^{T}(k) L x\left(k-h_{2}\right) .
\end{aligned}
$$

We take the forward difference of $V_{6}(k)$ as

$$
\begin{aligned}
\Delta V_{6}(k)= & y^{T}(k)\left[\frac{h_{1}^{4}}{4} F+\frac{\left(h_{2}-h_{1}\right)^{2}}{4} H\right] y(k) \\
& -\frac{h_{1}^{2}}{2} \sum_{i=-h_{1}} \sum_{j=k+i}^{k-1} y^{T}(j) F y(j) \\
& \frac{-1}{2} \sum_{i=k-h_{2}}^{k-h_{1}-1} \sum_{j=i}^{k-h_{1}-1} y^{T}(j) H y(j) .
\end{aligned}
$$

Furthermore, it follows from Lemma 2.4 that

$$
\begin{aligned}
& -\frac{h_{1}^{2}}{2} \sum_{i=-h_{1}}^{-1} \sum_{j=k+i}^{k-1} y^{T}(j) F y(j) \\
\leq & -\left[h_{1} x(k)-\left.\sum_{i=k-h_{1}}^{k-1} x(i)\right|^{T} F\left[h_{1} x(k)-\sum_{i=k-h_{1}}^{k-1} x(i)\right.\right.
\end{aligned}
$$




$$
\begin{aligned}
& -\frac{1}{2} \sum_{i=k-h_{2}}^{k-h_{1}-1} \sum_{j=i}^{k-h_{1}-1} y^{T}(j) H y(j) \\
= & -1 / 2 \sum_{i=k-h(k)}^{k-h_{1}-1} \sum_{j=i}^{k-h_{1}-1} y^{T}(j) H y(j) \\
& -1 / 2 \sum_{i=k-h_{2}}^{k-h(k)-1} \sum_{j=i}^{k-h(k)-1} y^{T}(j) H y(j) \\
& -1 / 2 \sum_{i=k-h_{2}}^{k-h(k)-1} \sum_{j=k-h(k)}^{k-h_{1}-1} y^{T}(j) H y(j) \\
\leq & -\left[\sum_{i=k-h(k)}^{k-h_{1}-1} \sum_{j=i}^{k-h_{1}-1} y(j)\right. \\
& -\left[\sum_{i=k-h_{2}}^{k-h(k)-1} \sum_{j=i}^{k-h(k)-1} y(j)\right. \\
= & -\left[x\left(k-h_{1}\right)-\psi(k)\right]^{2} H\left[x\left(k-h_{1}\right)-\psi(k)\right] \\
- & -[x(k-h(k))-\phi(k)]^{T} H[x(k-h(k))-\phi(k)] .
\end{aligned}
$$

Therefore, we obtain

$$
\begin{aligned}
& \Delta V_{6}(k) \leq y^{T}(k)\left[\frac{h_{1}^{4}}{4} F+\frac{\left(h_{2}-h_{1}\right)^{2}}{4} H\right] y(k) \\
& -\left[h_{1} x(k)-\sum_{i=k-h_{1}}^{k-1} x(i){ }^{T} F\left[h_{1} x(k)-\sum_{i=k-h_{1}}^{k-1} x(i)\right.\right. \\
& -\left[x\left(k-h_{1}\right)-\psi(k)\right]^{T} H\left[x\left(k-h_{1}\right)-\psi(k)\right] \\
& -[x(k-h(k))-\phi(k)]^{T} H[x(k-h(k))-\phi(k)] .
\end{aligned}
$$

It is obvious that

$$
\begin{aligned}
\Upsilon & \equiv x(k)-x\left(k-h_{1}\right)-\sum_{i=k-h_{1}}^{k-1} y(i)=0, \\
\Phi & \equiv x(k)-x\left(k-h_{2}\right)-\sum_{i=k-h_{2}}^{k-1} y(i)=0,
\end{aligned}
$$




$$
\begin{aligned}
\Psi & \equiv x(k)-x(k-h(k))-\sum_{i=k-h(k)}^{k-1} y(i)=0, \\
\Omega & \equiv x\left(k-h_{1}\right)-x\left(k-h_{2}\right)-\sum_{i=k-h_{2}}^{k-h_{1}-1} y(i)=0 .
\end{aligned}
$$

The following equations are true for any matrices with appropriate dimensions:

$$
\begin{aligned}
& {\left[2 x^{T}(k) C_{1}^{T}+2 y^{T}(k) C_{2}^{T}+2 x^{T}\left(k-h_{1}\right) C_{3}^{T}\right.} \\
& +2 x^{T}\left(k-h_{2}\right) C_{4}^{T}+2 x^{T}(k-h(k)) C_{5}^{T} \\
& +2\left(\sum_{i=k-h_{1}}^{k-1} y(i)\right)^{T} C_{6}^{T}+2\left(\sum_{i=k-h_{2}}^{k-1} y(i)\right)^{T} C_{7}^{T} \\
& +2\left(\sum_{i=k-h(k)}^{k-1} y(i)\right)^{T} C_{8}^{T}+2\left(\sum_{i=k-h_{2}}^{k-h_{1}-1} y(i)\right)^{T} C_{9}^{T} \\
& +2 \phi^{T}(k) C_{10}^{T}+2 \psi^{T}(k) C_{11}^{T}+2 f^{T}(k, x(k)) C_{12}^{T} \\
& \left.+2 g^{T}(k, x(k-h(k))) C_{13}^{T}\right] \times \Upsilon=0, \\
& {\left[2 x^{T}(k) D_{1}^{T}+2 y^{T}(k) D_{2}^{T}+2 x^{T}\left(k-h_{1}\right) D_{3}^{T}\right.} \\
& +2 x^{T}\left(k-h_{2}\right) D_{4}^{T}+2 x^{T}(k-h(k)) D_{5}^{T} \\
& +2\left(\sum_{i=k-h_{1}}^{k-1} y(i)\right)^{T} D_{6}^{T}+2\left(\sum_{i=k-h_{2}}^{k-1} y(i)\right)^{T} D_{7}^{T} \\
& +2\left(\sum_{i=k-h(k)}^{k-1} y(i)\right)^{T} D_{8}^{T}+2\left(\sum_{i=k-h_{2}}^{k-h_{1}-1} y(i)\right)^{T} D_{9}^{T} \\
& +2 \phi^{T}(k) D_{10}^{T}+2 \psi^{T}(k) D_{11}^{T}+2 f^{T}(k, x(k)) D_{12}^{T} \\
& \left.+2 g^{T}(k, x(k-h(k))) D_{13}^{T}\right] \times \Phi=0, \\
& {\left[2 x^{T}(k) E_{1}^{T}+2 y^{T}(k) E_{2}^{T}+2 x^{T}\left(k-h_{1}\right) E_{3}^{T}\right.} \\
& +2 x^{T}\left(k-h_{2}\right) E_{4}^{T}+2 x^{T}(k-h(k)) E_{5}^{T} \\
& +2\left(\sum_{i=k-h_{1}}^{k-1} y(i)\right)^{T} E_{6}^{T}+2\left(\sum_{i=k-h_{2}}^{k-1} y(i)\right)^{T} E_{7}^{T}
\end{aligned}
$$




$$
\begin{aligned}
& +2\left(\sum_{i=k-h(k)}^{k-1} y(i)\right)^{T} E_{8}^{T}+2\left(\sum_{i=k-h_{2}}^{k-h_{1}-1} y(i)\right)^{T} E_{9}^{T} \\
& +2 \phi^{T}(k) E_{10}^{T}+2 \psi^{T}(k) E_{11}^{T}+2 f^{T}(k, x(k)) E_{12}^{T} \\
& \left.+2 g^{T}(k, x(k-h(k))) E_{13}^{T}\right] \times \Psi=0, \\
& {\left[2 x^{T}(k) G_{1}^{T}+2 y^{T}(k) G_{2}^{T}+2 x^{T}\left(k-h_{1}\right) G_{3}^{T}\right.} \\
& +2 x^{T}\left(k-h_{2}\right) G_{4}^{T}+2 x^{T}(k-h(k)) G_{5}^{T} \\
& +2\left(\sum_{i=k-h_{1}}^{k-1} y(i)\right)^{T} G_{6}^{T}+2\left(\sum_{i=k-h_{2}}^{k-1} y(i)\right)^{T} G_{7}^{T} \\
& +2\left(\sum_{i=k-h(k)}^{k-1} y(i)\right)^{T} G_{8}^{T}+2\left(\sum_{i=k-h_{2}}^{k-h_{1}-1} y(i)\right)^{T} G_{9}^{T} \\
& +2 \phi^{T}(k) G_{10}^{T}+2 \psi^{T}(k) G_{11}^{T}+2 f^{T}(k, x(k)) G_{12}^{T} \\
& \left.+2 g^{T}(k, x(k-h(k))) G_{13}^{T}\right] \times \Omega=0 .
\end{aligned}
$$

From (3) and (4), we obtain for any scalars $\epsilon_{1}, \epsilon_{2}>0$,

$$
\begin{aligned}
& \epsilon_{1}\left(\alpha^{2} x^{T}(k) x(k)-f^{T}(k, x(k)) f(k, x(k))\right) \geq 0, \\
& \epsilon_{2}\left(\beta^{2} x^{T}(k-h(k)) x(k-h(k))\right. \\
& \left.-g^{T}(k, x(k-h(k))) g(k, x(k-h(k)))\right) \geq 0 .
\end{aligned}
$$

It follows form (15)-(36) that

$$
\Delta V(k) \leq \xi^{T}(k) \prod \xi(k),
$$

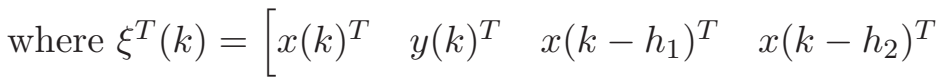

$x(k-h(k))^{T} \quad \sum_{i=k-h_{1}}^{k-1} y^{T}(i) \quad \sum_{i=k-h_{2}}^{k-1} y^{T}(i)$

$\sum_{i=k-h(k)}^{k-1} y(i)^{T} \quad \sum_{i=k-h_{2}}^{k-h_{1}-1} y^{T}(i) \quad \phi(k) \quad \psi(k)$

$f(k, x(k)) \quad g(k, x(k-h(k)))]$ and $\prod$ is defined in (10). By (37), if conditions (11)-(13) are true, then

$$
\Delta V(k)<-\omega\|x\|^{2},
$$

where $\omega>0$. This means that system (1)-(2) is asymptotically stable. The proof of theorem is complete. 
If $f(k, x(k))=g(k, x(k-h(k)))=0$ then system (1)-(2) reduces to the following system:

$$
\begin{aligned}
x(k+1) & =A x(k)+B x(k-h(k)), \\
x(s) & =\phi(s), \quad s \in\left\{-h_{2}, \ldots,-1,0\right\} .
\end{aligned}
$$

According to Theorem 3.1, we have the following Corollary 3.2 for the delayrange-dependent asymptotic stability criteria of system (39)-(40). We introduce the following notations for later use.

$$
\coprod=\left(\Omega_{k, l}\right)_{11 \times 11},
$$

where $\quad \Omega_{k, l}=\Omega_{l, k}^{T}=\Sigma_{k, l}, \quad k, l=1,2,3, \ldots, 11$, except $\Omega_{1,1}=\Sigma_{1,1}-\epsilon_{1} \alpha^{2} I$, $\Omega_{5,5}=\Sigma_{5,5}-\epsilon_{2} \beta^{2} I$.

Corollary 3.2. The system (39)-(40) is asymptotically stable, if there exist positive definite symmetric matrices $P, Q, R, S, T, U, V$,

$W, X, Y, Z$, any appropriate dimensional matrices $L^{w}, C^{j}, D^{j}, E^{j}$, $G^{j}, M, N, K, L, J, w=1,2,3, j=1,2, \ldots, 11$ such that the following symmetric linear matrix inequalities hold:

$$
\begin{gathered}
\amalg<0, \\
\left(\begin{array}{cc}
M & N \\
* & Y
\end{array}\right) \geq 0, \\
\left(\begin{array}{cc}
K & L \\
* & Z
\end{array}\right) \geq 0 .
\end{gathered}
$$

\section{Numerical Examples}

Example 4.1 Consider the system (1)-(2) with the following parameters which is considered in [16] and [24] :

$$
\begin{gathered}
A=\left(\begin{array}{cc}
0.80 & 0 \\
0.05 & 0.90
\end{array}\right), B=\left(\begin{array}{cc}
-0.10 & 0 \\
-0.20 & -0.10
\end{array}\right), \\
\alpha \geq 0, \quad \beta \geq 0 .
\end{gathered}
$$

By using the LMI Toolbox in MATLAB (with accuracy 0.01) for Theorem 3.1 to system (1)-(2) with (45)-(46), one can obtain the maximum upper bounds of the time delay under different values of $h_{1}$ as shown in Table 1 . We can see 
Table 1: Upper bounds of time delay $h_{2}$ for different conditions for Example 4.1.

\begin{tabular}{cccccc}
\hline \hline Method & $h_{1}$ & 2 & 6 & 10 & 14 \\
\hline$\alpha=0.1, \beta=0$ & & & & & \\
\hline Zhang et al. (2011) [24] & $h_{2}$ & 10 & 12 & 14 & 17 \\
Ramakrishnan and Ray (2013) $[16]$ & $h_{2}$ & 13 & 14 & 16 & 18 \\
Corollary 3.1 & $h_{2}$ & 21 & 23 & 23 & 24 \\
\hline$\alpha=0, \beta=0.1$ & & & & & \\
\hline Ramakrishnan and Ray (2013) $[16]$ & $h_{2}$ & 11 & 13 & 15 & 17 \\
Corollary 3.1 & $h_{2}$ & 19 & 24 & 23 & 22 \\
\hline$\alpha=0.1, \beta=0.1$ & & & & & \\
\hline Ramakrishnan and Ray (2013) $[16]$ & $h_{2}$ & 10 & 11 & 13 & 15 \\
Corollary 3.1 & $h_{2}$ & 16 & 19 & 22 & 21 \\
\hline
\end{tabular}

that our results in Theorem 3.1 are much less conservative than in [16] and [24].

Example 4.2 Consider the system (39)-(40) with the following parameters which is considered in [3], [5], [22] and [23] :

$$
A=\left(\begin{array}{cc}
0.80 & 0 \\
0.05 & 0.90
\end{array}\right), \quad B=\left(\begin{array}{cc}
-0.10 & 0 \\
-0.20 & -0.10
\end{array}\right) \text {. }
$$

By using the LMI Toolbox in MATLAB (with accuracy 0.01) for Corollary 3.2 to system (39)-(40) with (47), the maximum upper bounds $h_{2}$ for asymptotic stability of Example 4.2 is listed in the comparison in Table 2, for different values of $h_{1}$. We can see that our results in Corollary 3.2 are much less conservative than in [3], [5], [22] and [23].

Table 2: Upper bounds of time delay $h_{2}$ for different conditions for Example 4.2.

\begin{tabular}{cccccc}
\hline \hline Method & $h_{1}$ & 2 & 6 & 10 & 14 \\
\hline Fridman and Shaked (2005) [3] & $h_{2}$ & 13 & 15 & 19 & 23 \\
Gao and Chen (2007) [5] & $h_{2}$ & 14 & 15 & 18 & 22 \\
Zhang et al. (2010) [22] & $h_{2}$ & 15 & 17 & 21 & 25 \\
Zhang et al. (2011) [23] & $h_{2}$ & 15 & 17 & 21 & 25 \\
Corollary 3.2 & $h_{2}$ & $>16$ & $>18$ & $>22$ & $>26$ \\
\hline
\end{tabular}




\section{Conclusion}

In this paper, the problem of asymptotic stability analysis for discrete-time linear system with interval time-varying delay and nonlinear perturbations has been presented. The method combining augmented Lyapunov-Krasovskii functional, mixed model transformation, Jensen-type summation inequality and utilization of zero equation have been studied. New delay-range-dependent asymptotic stability criteria have been obtained and formulated in terms of LMIs. By comparing the proposed results with the results available in the existing literature, it is shown that the derived criteria are less conservative.

\section{Acknowledgments}

This work is supported by National Research Council of Thailand and Khon Kaen University, Thailand (Grant number 570021).

\section{References}

[1] B.T. Cui, M.G. Hua, Robust passive control for uncertain discrete-time system with time-varying delays, Chaos Solitons Fractals, 29, No. 2 (2006), 331-341. DOI : 10.1016/j.chaos.2005.08.039.

[2] M. Fang, Delay-dependent Stability analysis for discrete singular systems with time-varying delays, Acta Automat. Sinica, 36, No. 5 (2010), 751-755. DOI : 10.3724/SP.J.1004.2010.00751.

[3] E. Fridman, U. Shaked, Stability and guaranteed cost control of uncertain discrete delay systems, Internat. J. Control, 78, No. 4 (2005), 235-246. DOI : $10.1080 / 00207170500041472$.

[4] A. González, Robust stabilization of linear discrete-time systems with time-varying input delay, Automatica, 49, No. 9 (2013), 2919-2922. DOI : 10.1016/j.automatica.2013.05.031.

[5] H. Gao, T. Chen, New results on stability of discrete-time systems with time-varying state delay, IEEE Trans. Automat. Control, 52, No. 2 (2007), 328-334. DOI : 10.1109/TAC.2006.890320.

[6] K. Gu, V.L. Kharitonov, J. Chen, Stability of Time-Delay Systems, Birkhäuser Berlin, Germany (2003). 
[7] Y. He, M. We, J.H. She, G.P. Liu, Delay-dependent Lyapunov functional for stability of time-delay systems with polytopic-type uncertainties, IEEE Trans. Automat. Control, 49, No. 5 (2004), 828-832. DOI : 10.1109/TAC.2004.828317.

[8] X. Jiang, Q. Han, X. Yu, Stability criteria for linear discrete-time systems with interval-like timevarying delay, Proc. Amer. Math. Soc., 4, (2005), 2817-2822.

[9] J. Liu, J. Zhang, Note on stability of discrete-time time-varying delay systems, IET Control Theory Appl., 6, No. 2 (2012), 335-339. DOI : 10.1049/iet-cta.2011.0147.

[10] P.L. Liu, Further improvement on delay-range-dependent stability results for linear systems with interval time-varying delays, ISA Transactions, 52, No. 6 (2013), 725-729. DOI : 10.1016/j.isatra.2013.07.006.

[11] X. Liu, R. Martin, M. Wu, and M. Tang, Delay-dependent robust stabilization of discrete-time systems with time-varying delay, IEE Proc. Control Theory Appl., 152, (2006), 147-151.

[12] Z.X. Liu, S. Lü, S.M. Zhong, M. Ye, Stabilization analysis for discretetime systems with time delay, Appl. Math. Comput., 216, No. 7 (2010), 20242035. DOI : 10.1016/j.amc.2010.03.033.

[13] M.S. Mahmoud, N.B. Almutairi, Robust stability and stabilization methods for a class of nonlinear discrete-time delay systems, Appl. Math. Comput., 215, No. 12 (2010), 4280-4292. DOI : 10.1016/j.amc.2009.12.054.

[14] K. Mukdasai, Robust exponential stability for LPD discrete-time system with interval time-varying delay, J. Appl. Math., 2012, (2012), Article ID 237430, 13 pages. DOI : 10.1155/2012/237430.

[15] K. Mukdasai, P. Niamsup, Robust stability of discrete-time linear parameter dependent system with delay, Thai J. Math., Special Issue (Annual Meeting in Mathematics) (2010), 11-20.

[16] K. Ramakrishnan, G. Ray, Robust stability criteria for a class of uncertain discrete-time systems with time-varying delay, Appl. Math. Model., 37, No. 3 (2013), 1468-1479. DOI : 10.1016/j.apm.2012.03.045.

[17] J. Sun, G.P. Liu, J. Chen, D. Rees, Improved delay-range-dependent stability criteria for linear systems with time-varying delays, Automatica, 46, No. 2 (2010), 466-470. DOI : 10.1016/j.automatica.2009.11.002. 
[18] S. Udpin, P. Niamsup, Robust stability of discrete-time LPD neural networks with time-varying delay, Commun. Nonlinear Sci. Numer. Simul., 14, No. 11 (2009), 3914-3924. DOI : 10.1016/j.cnsns.2008.08.018.

[19] T. Wang, M.X. Xue, S.M. Fei, T. Li, Triple Lyapunov functional technique on delay-dependent stability for discrete-time dynamical networks, Neurocomputing, 122, (2013), 221-228. DOI : 10.1016/j.neucom.2013.05.039.

[20] Z.G. Wu, P. Shi, H. Su, J. Chu, Delay-dependent exponential stability analysis for discrete-time switched neural networks with timevarying delay, Neurocomputing, 74, No. 10 (2011), 1626-1631. DOI : 10.1016/j.neucom.2011.01.015.

[21] S.Y Xu, J. Lam, C.W. Yang, Quadratic stability and stabilization of uncertain linear discrete-time systems with state delay, Systems Control Lett., 43, No. 2 (2001), 77-84. DOI : 10.1016/S0167-6911(00)00113-4.

[22] W. Zhang, Q.Y. Xie, X.S. Cai, Z.Z. Han, New stability criteria for discretetime systems with interval time-varying delay and polytopic uncertainty, Latin American Applied Research, 40, No. 2 (2010), 119-124.

[23] W. Zhang, J. Wang, Y. Liang, Z.Z. Han, Improved delay-range-dependent stability criterion for discrete-time systems with interval time-varying delay, J. Inf. Comput. Sci., 14, No. 8 (2011), 3321-3328.

[24] W. Zhang, H.S. Su, Z.Z. Han, Robust stability for nonlinear discrete-time systems with interval time-varying delay, 2011 Third International Conference on Measuring Technology and Mechatronics Automation, (2011), 191-194. DOI : 10.1109/ICMTMA.2011.618.

[25] W.A. Zhang, L. Yu, Stability analysis for discrete-time switched timedelay systems, Automatica, 45, No. 10 (2009), 2265-2271. DOI : 10.1016/j.automatica.2009.05.027.

[26] Z.Q. Zuo, H.C. Li, Y.J. Wang, New criterion for finite-time stability of linear discrete-time systems with time-varying delay, J. Franklin Inst., 350, No. 9 (2013), 2745-2756. DOI : 10.1016/j.jfranklin.2013.06.017. 
